\title{
Nanoscale Heterogeneous Structure of Ionic Liquid as Revealed by Magnetic Field Effects
}

\author{
Masanobu Wakasa,* Tomoaki Yago, and Atom Hamasaki ${ }^{\dagger}$ \\ Department of Chemistry, Graduate School of Science and Engineering, Saitama University, \\ 255 Shimo-okubo, Sakura-ku, Saitama-shi, Saitama 338-8570, Japan
}

Received: June 13, 2009; Revised Manuscript Received: July 8, 2009

\begin{abstract}
Large magnetic field effects (MFEs) observed for photoinduced hydrogen abstraction reaction between benzophenone and thiophenol in an ionic liquid $(N, N, N$,-trimethyl- $N$-propylammonium bis(trifluoromethanesulfonyl)amide, TMPA TFSA) are analyzed by using the stochastic Liouville equation for the first time. The sphere cage model can well reproduce the observed MFEs and the nanoscale heterogeneous structure with a cage radius of $1.8 \pm 0.3 \mathrm{~nm}$, and an effective viscosity in the cage of $1-2 \mathrm{cP}$ is found to be formed in TMPA TFSA.
\end{abstract}

\section{Introduction}

Ionic liquids (ILs) are one of the most promising new classes of solvents in green chemistry, electrochemistry, and nanochemistry. ${ }^{1-7}$ Their exceptional combination of properties-nonvolatility, noncorrosiveness, nonflammability, stability to air and moisture, and designability-provides new environments for chemical reactions. Recently, some novel chemical and photochemical reactions in ILs have been reported, ${ }^{7,8}$ which may be due to their structural heterogeneity. Although theoretical and spectroscopic studies suggest that ILs have a heterogeneous structure as a result of strong ionic interactions and the aggregation of the nonpolar parts of ionic molecules, ${ }^{9-12}$ the details are still unclear. Thus, understanding the nature of the heterogeneity of ILs is very important. If this is clarified, ILs may supersede conventional solvents.

In 2007, we studied the magnetic field effects (MFEs) on photochemical reactions in ILs and found large MFEs on the radical pair (RP) generated by the photoinduced hydrogen abstraction reaction between benzophenone (BP) and thiophenol $(\mathrm{PhSH})$ in an IL of $N, N, N$,-trimethyl- $N$-propyl-ammonium bis(trifluoromethanesulfonyl) amide (TMPA TFSA). ${ }^{13}$ In the previous paper, we suggested a cage effect caused by the heterogeneity of the IL as a qualitative explanation for such large MFEs on the RP. In RPs generated by photochemical reactions, the unpaired electron spins on each radical are coupled, giving two different spin states: singlet (S) and triplet (T). According to the Pauli principle, singlet RPs can react to form a recombination product, whereas triplet RPs cannot react in each other but form an escaped radical. Magnetic fields interact with these spins and affect the reaction of the RPs without changing other parameters such as the reaction rate of singlet RPs, activation barrier, and diffusion motion of the radicals. Since the interaction between magnetic fields and spins can be described by quantum chemistry, MFE studies on RPs provide valuable information on their kinetics and dynamics,

\footnotetext{
* To whom correspondence should be addressed. E-mail: mwakasa@ chem.saitama-u.ac.jp.

$\dagger$ Present address: Department of Chemistry, Faculty of Science, Shinshu University, 3-1-1 Asahi, Matsumoto, Nagano 390-8621, Japan.
}

\section{SCHEME 1: Cage Model Employed for the SLE Analysis $^{a}$}

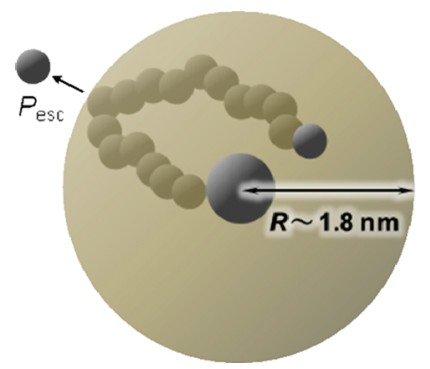

a One radical is fixed to the center of the sphere cage, while the other radical diffuses freely in the cage. The radical pair is recombined at the contact or is allowed to escape with a certain probability $\left(P_{\text {esc }}\right)$ from the cage at the interface.

and in particular on aspects of the environment around RPs such as heterogeneity, domain, and cage size. ${ }^{14,15}$ In this paper, we describe the first numerical analysis of the MFEs observed in the IL by using the stochastic Liouville equation (SLE). Here, we report that the sphere cage model can well reproduce the observed MFEs and the nanoscale heterogeneous structure with a cage radius $(R)$ of $1.8 \pm 0.3 \mathrm{~nm}$ and an effective viscosity $(\eta)$ in the cage of $1-2 \mathrm{cP}$ is formed in TMPA TFSA.

\section{Methods}

We used the Pedersen-Freed type SLE, ${ }^{16}$ in which the spin Hamiltonian consists of the exchange interaction $(J)$, the hyperfine interaction, and the Zeeman interactions caused by the magnetic field. The radical diffusion, which is assumed to be simple Brownian motion, is treated with the finite difference technique and mutual diffusion constant. Details of the SLE analysis are described in our previous report ${ }^{17}$ and in the Supporting Information. Since the cage effects have been suggested from the much larger MFEs than that usually found in isotropic liquids, ${ }^{13}$ in this study, we employed the sphere cage model shown schematically in Scheme 1, which has been applied to micellar solutions. ${ }^{18-20}$ To the best of our knowledge, 


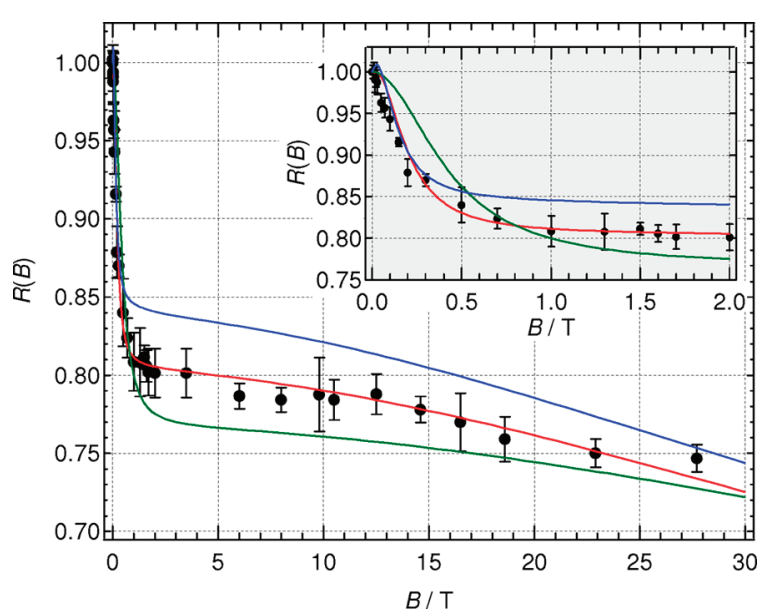

Figure 1. Magnetic field dependence of the relative yield $(R(B \mathrm{~T}))$ of the escaped radical calculated by the SLE analysis with the parameters of $P_{\text {esc }}=8.3 \times 10^{-4}$ and $R=1.3$ (green), 1.8 (red), and $2.3 \mathrm{~nm}$ (blue). Filled circles are the corresponding experimental results obtained in TMPA TFSA. The inset shows details of the data at lower values of the magnetic field.

there is no report on the shape of domain in ILs. For the first attempt, we assumed that the cage is spherical and successfully reproduced the experimental results by the SLE.

\section{Results and Discussion}

The MFEs observed for the hydrogen abstraction reaction between $\mathrm{BP}$ and $\mathrm{PhSH}$ in TMPA TFSA have the following characteristics: ${ }^{13}$ (1) The escape yield of the benzophenone ketyl radical generated from the hydrogen abstraction reaction decreased with increasing magnetic field strength $(B)$ in the range of $0 \mathrm{~T}<B \leq 2 \mathrm{~T}$. (2) The decease was almost saturated for $2 \mathrm{~T}<B \leq 10 \mathrm{~T}$. (3) At much higher fields $(10 \mathrm{~T}<B \leq 28$ $\mathrm{T})$, the yield deceased again with increasing $B$, resulting in a $25 \%$ decrease at $28 \mathrm{~T}$. This magnetic field dependence was analyzed by the SLE in which the spin dynamics, spin relaxations, diffusion, and reactive dynamics were taken into account simultaneously. In this study, we employed the sphere cage model, as shown in Scheme 1. In this cage, contact RPs are produced through the hydrogen abstraction reaction of BP from $\mathrm{PhSH}$. Radicals are diffused from the contact RP, producing separate RPs. The separated RPs are re-encountered to form the contact RP. In the cage, these processes are repeated until the RPs are recombined at the contact or escape from the cage at the boundary with a certain probability. For simplicity, we assume one radical is fixed to the center of the sphere cage while the other radical diffuses freely in the cage.

The fitting parameters are the cage radius $(R)$, escape probability $\left(P_{\text {esc }}\right)$ of radicals from the cage, mutual diffusion coefficient $(D)$ for the translational RP diffusion in the cage, and correlation time $(\tau)$ for the radicals. Figure 1 shows the typical magnetic field dependences $(R(B \mathrm{~T})=Y(B \mathrm{~T}) / Y(0 \mathrm{~T}))$ of the yields $(Y)$ of the escaped radicals obtained by the SLE analysis together with the experimental results. ${ }^{13}$ The experimental results are well-reproduced by the SLE simulation with parameter values of $R=1.8 \mathrm{~nm}, P_{\mathrm{esc}}=8.3 \times 10^{-4}, D=1.6$ $\times 10^{-9} \mathrm{~m}^{2} \mathrm{~s}^{-1}$, and $\tau=5 \mathrm{~ns}$ for both radicals. In addition, we include an additional correlation time of $\tau=60$ fs to account for the MFEs observed in the region of $B>10 \mathrm{~T}$. This value is considered to be associated with the vibrational motion of the solute molecule. ${ }^{21}$

The SLE analysis indicates that the main features of the observed MFEs can be explained by the $\Delta g$ mechanism $^{15}$ combined with $B$-dependent spin relaxations. The SLE analysis also reveals that the transverse spin relaxation $\left(\mathrm{S}-\mathrm{T}_{0}\right.$ relaxation) contributes to the saturation of the MFEs in the region of $B<$ $5 \mathrm{~T}$, while the longitudinal spin relaxation $\left(\mathrm{S}-\mathrm{T}_{ \pm}, \mathrm{T}_{0}-\mathrm{T}_{ \pm}\right.$ relaxation) is responsible for the MFEs observed in the region of $B>10 \mathrm{~T} .{ }^{15}$ These spin relaxations are caused by the large anisotropy of the $g$ value in the phenylthiyl radical.

In the simulations, the $D$ value was somewhat arbitrary. The $D$ value used in the simulations yielded an effective viscosity in the cage of $1 \mathrm{cP} .^{22}$ When a $D$ value of $8.1 \times 10^{-10} \mathrm{~m}^{2} \mathrm{~s}^{-1}$ was used for $2 \mathrm{cP}$, similar aspects of the SLE simulation (Supporting Information) were obtained with a slightly small $P_{\text {esc }}$ value of $8.0 \times 10^{-4}$. Thus, we concluded that the effective viscosity in the cage is $1-2 \mathrm{cP}$. In our previous paper, we estimated that the viscosity in the cage should be smaller than $5 \mathrm{cP}$ from the results of the MFEs observed for the same reaction in alcoholic solutions. ${ }^{17,23}$ These values are 1 order of magnitude smaller than the macroscopic viscosity of TMPA TFSA. Wishart and Castner et al. reported that there are several microviscosities for ILs ranging from orders of magnitude smaller than the macroscopic viscosities to viscosities similar to or larger than the macroscopic viscosities. ${ }^{24}$ The present results also support the existence of several microviscosities in TMPA TFSA.

From the SLE analysis, we estimated the $R$ value to be 1.8 $\pm 0.3 \mathrm{~nm}$ under the assumption that the cage is spherical. When $R$ is smaller than $1.3 \mathrm{~nm}, \mathrm{~S}-\mathrm{T}$ conversions by the $\Delta g$ mechanism and spin relaxation are not efficient in the region of $B<1 \mathrm{~T}$, since $J$ has a significant magnitude in the overall cage. When $R$ is larger than $2.3 \mathrm{~nm}$, there is enough space for $\mathrm{S}-\mathrm{T}$ conversions in the cage, while the re-encounter probabilities of RPs are reduced, causing the magnitude of the MFEs to be small.

The good agreement between experiment and analysis leads to the conclusion that a nanoscale heterogeneous structure with $R=1.8 \pm 0.3 \mathrm{~nm}$ and $\eta=1-2 \mathrm{cP}$ (in the cage) is formed in TMPA TFSA. In this heterogeneous structure, chemical reactions proceed efficiently. This conclusion is consistent with recent reports on the bimolecular electron transfer reaction system: back electron transfer reactions were enhanced by the cage effect in ILs. ${ }^{8}$ The present results may also be relevant to explain the efficient formation of nanoparticles in ILs. ${ }^{7}$ Regarding the origin of the heterogeneous structure, molecular simulations ${ }^{10-12}$ suggest that the alkyl side chains in ILs are aggregated, forming the isolated domain structures that may work as heterogeneous structures. The TMPA TFSA used in the present study does not have a long enough alkyl side chain $\left(\mathrm{C}_{3}\right)$ to form a heterogeneous structure with $R=1.8 \mathrm{~nm}$. Thus, the all-alkyl part of the ammonium-based cation may contribute to the large structure. To clarify this point, further studies with other kinds of ILs are now in progress.

Acknowledgment. This work was partially supported by a Grant-in-Aid for Scientific Research (Nos. 20081005 and 2003002) in the Priority Areas "Science of Ionic Liquids" (No. 452) and "High Field Spin Science in 100 T" (No. 451) from the Ministry of Education, Culture, Sports Science, and Technology (MEXT) of Japan.

Supporting Information Available: Details of the SLE analysis. This material is available free of charge via the Internet at http://pubs.acs.org.

\section{References and Notes}

(1) Seddon, K. R. J. Chem. Technol. Biotechnol. 1997, 68, 351-356.

(2) Welton, T. Chem. Rev. 1999, 99, 2071-2083. 
(3) Wasserscheid, P.; Keim, W. Angew. Chem., Int. Ed. 2000, 39, 37723789.

(4) Sheldon, R. Chem. Commun. 2001, 2399-2407.

(5) Dupont, J.; de Souza, R. F.; Suarez, P. A. Z. Chem. Rev. 2002, 102, 3667-3692.

(6) Ohno, H. Electrochemical Aspects of Ionic Liquids; John Wiley \& Sons, Inc.: Hoboken, NJ, 2005.

(7) Antonietti, M.; Kuang, D.; Smarsly, B.; Zhou, Y. Angew. Chem., Int. Ed. 2004, 43, 4988-4992.

(8) Paul, A.; Samanta, A. J. Phys. Chem. B 2007, 111, 1957-1962.

(9) Iwata, K.; Okajima, H.; Saha, S.; Hamaguchi, H. Acc. Chem. Res. 2007, 40, 1174-1181.

(10) Wang, Y.; Jiang, W.; Yan, T.; Voth, G. A. Acc. Chem. Res. 2007, $40,1193-1199$

(11) Hardacre, C.; Holbrey, J. D.; Nieuwenhuyzen, M.; Youngs, T. G. A. Acc. Chem. Res. 2007, 40, 1146-1155.

(12) Pádua, A. A.; Gomes, M. F. C.; Lopes, J. N. A. C. Acc. Chem. Res. 2007, 40, 1087-1096.

(13) (a) Wakasa, M. J. Phys. Chem. B 2007, 111, 9434-9436. (b) Hamasaki, A.; Yago, T.; Takamasu, T.; Kido, G.; Wakasa, M. J. Phys. Chem. B 2008, 112, 3375-3379.

(14) Nagakura, S.; Hayashi, H.; Azumi, T. Dynamic Spin Chemistry; Kodansha-Wiley: Tokyo, New York, 1998.

(15) Hayashi, H. Introduction to Dynamic Spin Chemistry; World Scientific: Singapore, 2004.
(16) (a) Pedersen, J. B.; Freed, J. H. J. Chem. Phys. 1973, 58, 2746-2762. (b) Pedersen, J. B.; Freed, J. H. J. Chem. Phys. 1973, 59, 2869-2885.

(17) Hamasaki, A.; Yago, T.; Wakasa, M. J. Phys. Chem. B 2008, 112, 14185-14192.

(18) (a) Tarasov, V. F.; Ghatlia, N. D.; Buchachenko, A. L.; Turro, N. J. J. Am. Chem. Soc. 1992, 114, 9517-9526. (b) Tarasov, V. F.; Ghatlia, N. D.; Avdievich, N. I.; Shkrob, I. A.; Buchachenko, A. L.; Turro, N. J. J. Am. Chem. Soc. 1994, 116, 2281-2291. (c) Tarasov, V. F.; Bagranskaya, E. G.; Shkrob, I. A.; Avdievich, N. I.; Ghatlia, N. D.; Lukzen, N. N.; Turro, N. J.; Sagdeev, R. Z. J. Am. Chem. Soc. 1995, 117, 110-118.

(19) (a) Shushin, A. I.; Pedersen, J. B.; Lolle, L. I. Chem. Phys. 1994, 188, 1-17. (b) Christensen, M.; Pedersen, J. B. Chem. Phys. 2003, 295, $235-241$.

(20) Okazaki, M.; Toriyama, K. J. Phys. Chem. 1995, 99, 17244-17250.

(21) Spin relaxations caused by molecular vibrational motions have been reported in micellar solutions. See ref 14.

(22) The radii of the benzophenone ketyl and phenylthiyl radicals are estimated to be 0.4 and $0.2 \mathrm{~nm}$, respectively. ${ }^{17}$ These values were used to estimate the viscosity in the cage with the Stokes-Einstein equation.

(23) From the experimental observation that MFEs are quenched by the heavy atom effect in high-viscosity solvents, the viscosity in the cage was estimated to be lower than $5 \mathrm{cP}$. See ref 17 .

(24) Funston, A. M.; Fadeeva, T. A.; Wishart, J. F.; Castner, E. W., Jr. J. Phys. Chem. B 2007, 111, 4963-4977.

JP905543K 